\title{
Left atrial thrombus despite continuous oral anticoagulant therapy
}

\author{
Ivancikova M, Sipka J, Skamla M, Kaliska G \\ Department of arrhythmology, The Middle Slovak Institute for Cardiovascular Diseases, Banská Bystrica, Slovakia
}

\begin{abstract}
Ivancikova M, Sipka J, Skamla M, Kaliska G. Left atrial thrombus despite continuous oral anticoagulant therapy. Cardiology Lett. 2020;29(4):256-258

Abstract. Left atrial thrombus may be detected by transoesophageal echocardiography in patients with atrial fibrillation despite continuous anticoagulation therapy. We would like to introduce a case report of a patient treated in our hospital, in whom ischemic stroke occurred despite effective anticoagulant treatment. Fig. 3, Ref. 11, on-line full text (Free, PDF) www.cardiologyletters.sk

Key words: left atrial appendage - thrombus - transoesophageal echocardiography - anticoagulation therapy
\end{abstract}

Left atrial appendage thrombus (LAT) is known as a sequelae of atrial fibrillation (AF) and atrial flutter (AFL) and is a major source of thromboembolism resulting in stroke. Transoesophageal echocardiography (TEE) is the gold standard for evaluating the presence of thrombus (1). Clinical risk factors include hypertension, congestive heart failure, age, female sex, structural heart disease or cardiomyopathy, use of anti-arrhythmic drugs, persistent versus paroxysmal AF, higher $\mathrm{CHA}_{2} \mathrm{DS}_{2}$ VASc score (2). Biomarker and imaging modalities that can predict resistant LAT include a BNP $>75 \mathrm{pg} / \mathrm{mL}$, increased d-dimer level, depressed ejection fraction, enlarged left atrial size, low left atrial appendage (LAA) velocities, and spontaneous echo contrast (SEC) detected within LAA $(3,4)$. In an observational study of patients undergoing TEE after a minimum of 3 weeks of uninterrupted warfarin prior to $\mathrm{DCCV}$ of $\mathrm{AF}$, the rate of LAT detection was from 6.13 to $7.7 \%(5,6)$. Even with the use of $\geq 4$ weeks of direct oral anticoagulant (DOAC) therapy, the incidence of LAT detection by TEE prior to the catheter ablation of AF or AFL is as high as 4.4\% (7). Currently, there are no established guidelines on appropriate management of left atrial thrombus found in patients who have already been on continuous anticoagulation therapy.

\section{Case report}

Our case report is about a 72-year-old female patient with arterial hypertension, long persistent atrial fibrillation

From Department of arrhythmology, The Middle Slovak Institute for Cardiovascular Diseases, Banská Bystrica, Slovakia

Manuscript received 14 May 2020; accepted for publication 21 May 2020 (diagnosed in 2016), initially on anticoagulation therapy with antagonist of vitamin K. For symptomatic 2nd. degree AV block 2:1 in 2013 she underwent implantation of a double chamber pacemaker. In 8/2018 she had a worsening of her clinical condition in terms of stroke, with right-sided hemiplegia and equilateral hemianopsia. Ischaemic stroke occurred at therapeutic warfarin level (INR at admission 2.48). Subsequently, warfarin was changed to apixaban $5 \mathrm{mg}$ twice a day. In 10/2018 (2 months after stroke), a TEE was performed. Despite 2 months with apixaban treatment, we verified a thrombus in the left atrial appendage (Figure 1). LAA flow velocity was $16-19 \mathrm{~cm} / \mathrm{s}$, which means severe LAA dysfunction. It is known that LAA dysfunction (with low flow velocity) is a predictor of thrombus formation and thromboembolic events. Because of this (stroke and LAA thrombus despite anticoagulation and severe LAA dysfunction), we decided to add acetylsalicylic acid to apixaban. After four weeks of dual therapy (aspirin + apixaban), TEE was performed with confirmation of spontaneous echo contrast of the 3rd degree, without the presence of thrombus in LAA. Due to the history of the past stroke despite continuous oral anticoagulant therapy, we decided to perform transcatheter left atrial appendage closure (LAAO). In February 2019 she underwent the procedure without complications: subsequently she was treated with apixaban and acetylsalicylic acid for three months after the procedure. During the first outpatient visit in May 2019 (3 months after LAAO), she underwent TEE examination with the evidence of the correct position of the occluder in the left appendage (Figure 2).

Address for correspondence: MUDr. Mária Ivančíková, Odd. Arytmií, SÚSCCH, a. s., Cesta k nemocnici 1, 97401 Banská Bystrica, Slovenská republika, e-mail: marivancikova@gmail.com 


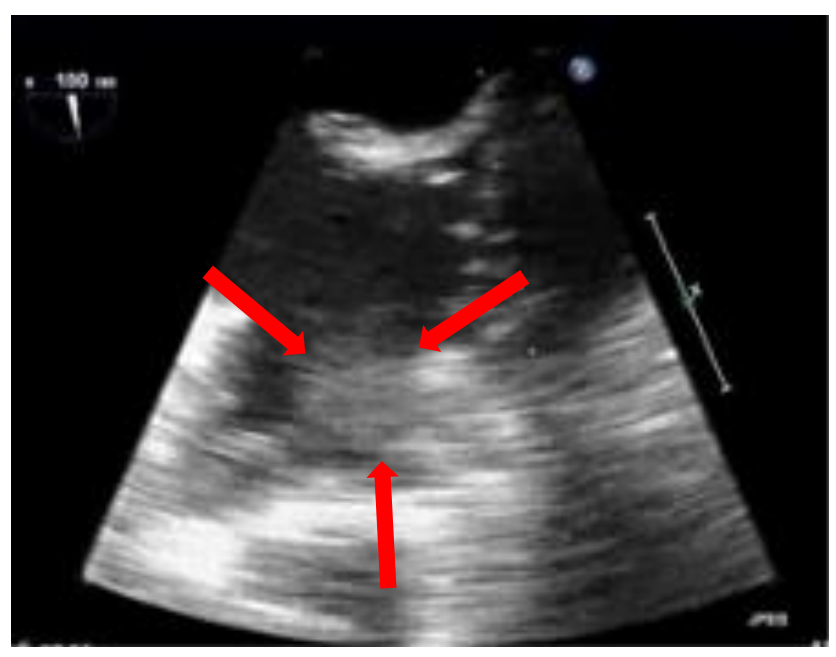

Figure 1 Thrombus in LAA

In a control laboratory sample, a decrease of haemoglobin was recorded (from $116 \mathrm{~g} / \mathrm{L}$ to $86 \mathrm{~g} / \mathrm{L}$ ). The character of anaemia was microcytic hypochromic. Because of the well-positioned occluder with no leaks, apixaban was stopped with the continuation of acetylsalicylic acid. Colonofibroscopy and gastrofibroscopy was performed, but no source of bleeding was elucidated. During the next inspection in December 2019 (7 months after apixaban withdrawal) TTE was performed, with the finding of fixed mass on the ventricular electrode, in the area of the right atrium, size $11 \times 16 \mathrm{~mm}$ - susp. thrombus

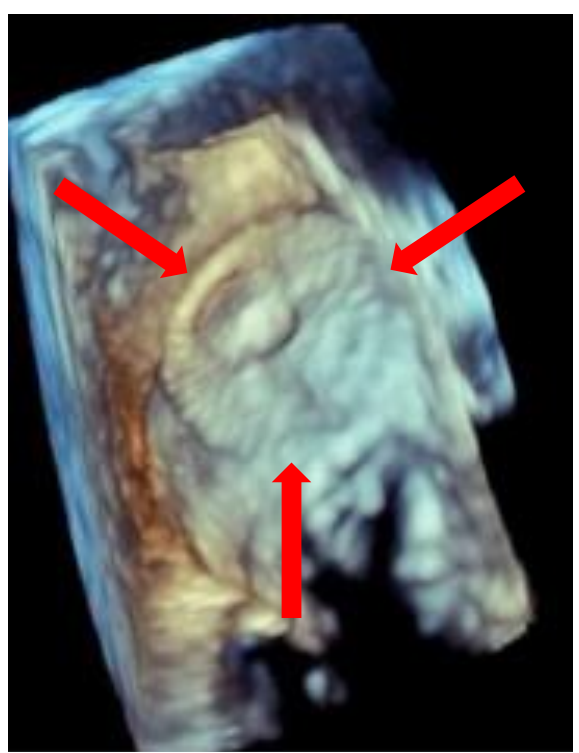

Figure 2 The correct postition of occluder in 3D TEE ( 3 months after LAAO)
(Figure 3). That is why apixaban was added to acetylsalicylic acid. Moderate anaemic syndrome persisted in the control blood count. In February 2020, control TTE was performed with confirmation of mass regression to $16 \times 6 \mathrm{~mm}$. Because of the asymptomatic condition and evident mass regression, we decided to continue with apixaban monotherapy. A not entirely clear echocardiographic finding on the electrode remains in our further observation.

\section{Discussion}

Optimal management of patients diagnosed with LAA thrombosis while taking an OAC is not well established. Evidence-based data on the effectiveness of NOAC for LAA thrombus resolution in patients previously taking a VKA is scarce, but switching from VKA to a NOAC was the most reported strategy $(8,9,10)$. There is lack of data informing on the optimal management of AF patients with persistent LAA thrombus despite optimized OAC therapy. A single publication described the resolution of warfarin - and dabigatran - resistant thrombus by apixaban (11). If the thrombus was still present after a course of OAC, the adopted strategy was another change of NOAC or addition of either antiplatelet agent or LMWH to the current treatment. In this case, dual treatment with apixaban and aspirin led to LAA thrombus resolution. Because of 'malignant' LAA with severe dysfunction and high risk of repeated thrombus formation, we decided to perform LAA occlusion in the non-pharmacological prevention of thromboembolism in AF.

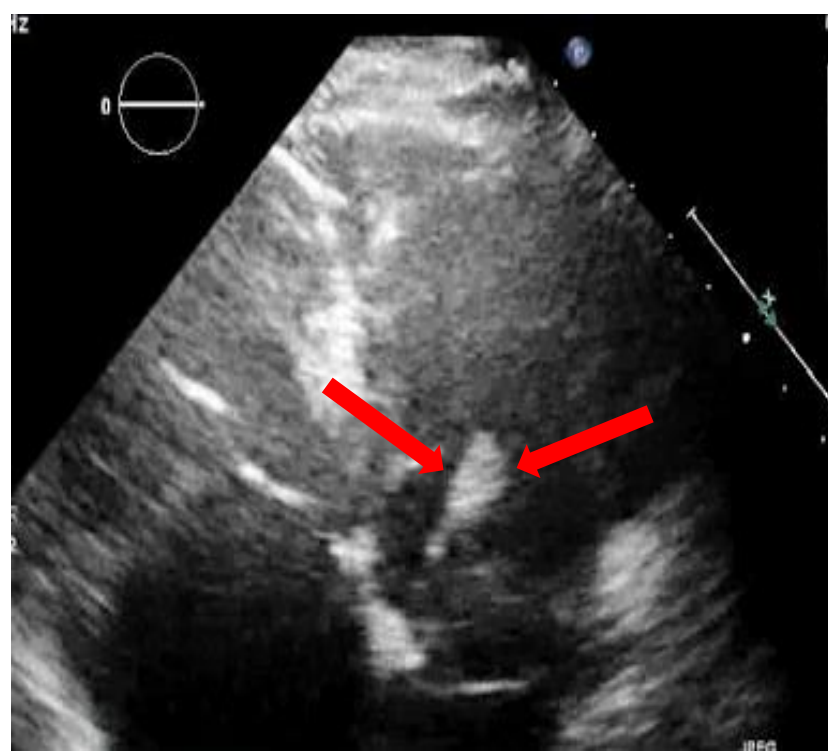

Figure 3 Susp. thrombus of pacemaker lead in right atrium (modified 4 chamber view) 


\section{Conclusion}

There are no established guidelines on the appropriate management of LAT found in patients who have already been on continuous anticoagulation therapy. Adding an antiplatelet agent to oral anticoagulation treatment can also be an option, as shown in our case report. Because of severe LAA dysfunction, there is still a substantial risk of repeated thrombus formation in LAA. Even dual therapy led to thrombus resolution; its long-term treatment is problematic because of high risk of bleeding. As shown in our case, LAA occlusion seems to be a reasonable option in this group of patients with 'malignant' LAA.

\section{References}

1. Al-Saady NM, Obel OA, Camm AJ. Left atrial appendage: structure, function, and role in thromboembolism. Heart 1999; 82:547854.

2. WU M, Gabriels J, Khan M, et al. Left atrial thrombus and dense spontaneous echocardiographic contrast in patients on continuous direct oral anticoagulant therapy undergoing catheters ablation of atrial fibrillation: comparison of dabigatran, rivaroxaban, and apixaban. Heart Rhythm 2018;15:496-502.

3. Calvo N, Mont L, Vidal B, et al. Usefulness of transoesophageal echocardiography before circumferential pulmonary vein ablation in patients with atrial fibrillation: is it really mandatory? Europace 2011;13:486-491.

4. Nishikii-Tachibana M, Murakoshi N, Seo Y, et al. Prevalence and clinical determinants of left atrial appendage thrombus in pa- tients with atrial fibrillation before pulmonary vein isolation. Am J Cardiol 2015;116:1368-1373.

5. Seidl L, Rameken M, Drogenmuller A, et al. Embolic events in patients with atrial fibrillation and effective anticoagulation: value of transesophageal echocardiography to guide direct-current cardioversion. Final results of the Ludwigshafen observational cardioversion study. J Am Coll Cardiol 2002;39:1436-1442.

6. Cresti A, Garcia-Fernández MA, Siievert H, et al. Prevalence of extra-appendage thrombosis in non-valvular atrial fibrillation and atrial flutter in patients undergoing cardioversion: a large transoesophageal echo study. Euro Intervention. 2019;15:225230.

7. Frenkel D, AL-Kazaz M, Markowitz SM, et al. Prevalence of left atrial thrombus by transesophageal echocardiography: a comparison of continuous non-vitamin $\mathrm{k}$ antagonist oral anticoagulant versus warfarin therapy in patients undergoing catheter ablation for atrial fibrillation. JACC Clin Electrophysiol 2016;2:295-303.

8. Lip GY, Hammerstingl C, Marin F, et al. Left atrial thrombus resolution in atrial fibrillation or flutter: results of a prospective study with rivaroxaban (X-TRA) and a retrospective observational registry providing baseline data (CLOT-AF) Am Heart J 2016;178:126-134.

9. Okada T, Takaekou Y, Idei N, et al. Resolution of left atrial appendage thrombus with apixaban in a patient with heart failure. Intern Med 2017;56:2891-2894.

10. Miwa Y, Minamishima T, Sato T, et al. Resolution of a warfarin and dabigatran-resistant left atrial appendage thrombus with apixaban. J Arrhythm 2016;32:233-2335.

11. Valero E, Santas E, Nunez J. Thrombolytic action of apixaban on intra-atrial thrombus developed after previous treatment with warfarin: a case report. Med Princ Pract 2016;25:491-493. 